\title{
Systemic Air Embolism
}

\author{
Jonathan Lorenz, M.D. ${ }^{1}$
}

Systemic air embolism is a rare but potentially devastating complication of percutaneous chest biopsy that requires immediate diagnosis and rapid initiation of aggressive, noninvasive rescue therapy to prevent death or permanent neurologic sequelae. Radiologists focused on the most common complications of chest biopsy such as pneumothorax and parenchymal hemorrhage run the risk of failing to quickly diagnose and effectively treat systemic air embolism. Furthermore, the low incidence of symptomatic air embolism may lead to complacency and failure to prepare proper institutional procedures for the effective treatment. This case reviews the incidence, postulated mechanisms, and treatment of systemic air embolism caused by percutaneous chest biopsy.

\section{CASE REPORT}

A 62-year-old man presented to a primary care clinic with a history of recent weight loss and a cough, productive of blood-tinged sputum. A computed tomography (CT) scan (Fig. 1) showed a solitary mass in the right middle lobe and he was referred to the interventional radiology service for percutaneous chest biopsy.

No sedation was performed during percutaneous chest biopsy. Oxygen saturation, heart rate, cardiac rhythm, and blood pressure were monitored throughout the procedure. The patient was placed supine on the CT table, and a 19-gauge (19G) introducer needle was advanced with a single pass into the lesion utilizing periodic, focused CT scans for guidance (Fig. 2). The patient was instructed to suspend respiration during each manipulation of the introducer and biopsy needles. After the first aspiration biopsy sample was obtained, the patient became unresponsive and a full code was called. During the code, the patient was intubated and initially bag ventilated with $100 \%$ oxygen by non-rebreather mask followed by mechanical ventilation.
An immediate postbiopsy head CT (Fig. 3) showed bubbles of intracranial air affecting the anterior and middle cerebral circulations. The patient eventually underwent treatment in a hyperbaric oxygen chamber and recovered with only mild, persistent left-sided weakness.

\section{DISCUSSION}

\section{Incidence}

Perhaps more surprising than the occasional reported case of systemic air embolism is the extremely low incidence of this complication in large, retrospective studies of thousands of patients that underwent percutaneous chest biopsy. ${ }^{1-3}$ Furthermore, a review of limited studies suggests that systemic air embolism is actually asymptomatic in the majority of cases. This low percentage of symptomatic cases is unexpected because less than $1 \mathrm{~mL}$ of air injected into a pulmonary vein can be fatal if embolization to the coronary circulation occurs. Symptomatic cases of systemic air embolism are usually recognized immediately by manifestations of mental status changes and cardiopulmonary compromise. Occasionally, onset of these symptoms occurs in delayed fashion, after movement of the patient or even after transfer from the radiology department. Although the incidence of cases of clinically manifested systemic air embolism is less than $0.1 \%$ in large retrospective studies and literature reviews, the incidence of all cases - both symptomatic and asymptomatic - has not been adequately assessed. Imaging following chest biopsy varies from radiography to computed tomography $(\mathrm{CT})$ localized to the biopsy site to complete chest CT. Performance of complete chest CT scans following biopsy captures some asymptomatic patients and raises the reported incidence to $0.4 \% .{ }^{4}$ Cross-sectional imaging

\footnotetext{
${ }^{1}$ Department of Radiology, The University of Chicago, Chicago, Illinois.

Address for correspondence and reprint requests: Jonathan Lorenz, M.D., Associate Professor of Radiology, Department of Radiology, The University of Chicago, 5841 S. Maryland Avenue, MC 2026, Chicago, IL 60637 (e-mail: jlorenz@radiology.bsd.uchicago.edu).

Complications in Interventional Oncology; Guest Editor, Robert J.
}

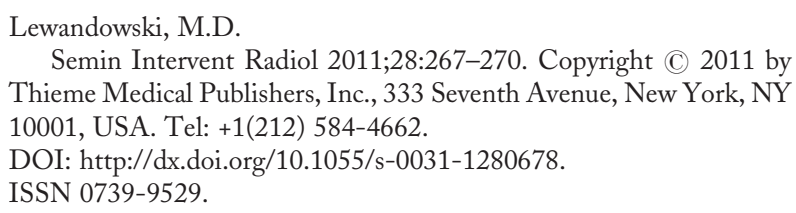
Thieme Medical Publishers, Inc., 333 Seventh Avenue, New York, NY 10001, USA. Tel: +1(212) 584-4662.

DOI: http://dx.doi.org/10.1055/s-0031-1280678. ISSN 0739-9529. 


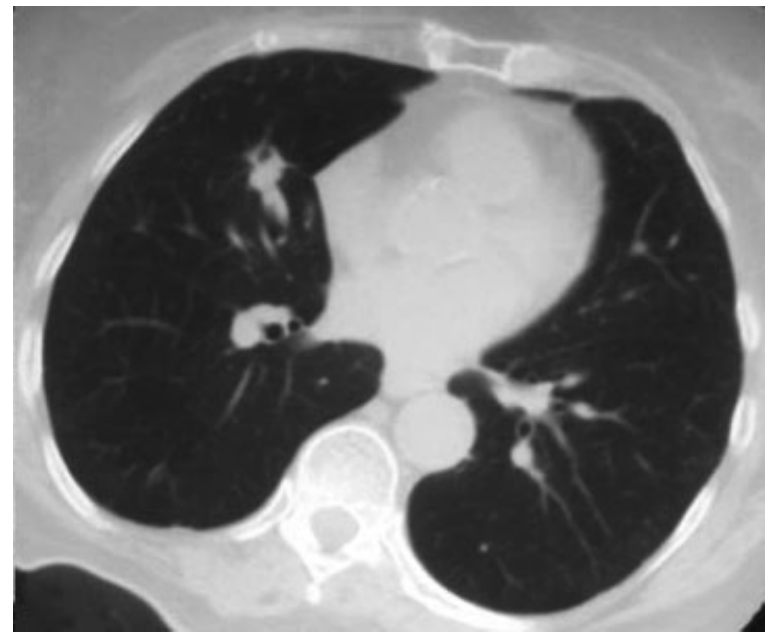

Figure 1 A 62-year-old man with a history of weight loss and a cough productive of blood-tinged sputum. Preliminary computed tomography scan shows a $1.5 \mathrm{~cm}$ mass in the right middle lobe abutting a pulmonary venous branch.

of the head after chest biopsy would likely yield additional asymptomatic cases. Therefore, the incidence of symptomatic cases is most likely less than $20 \%$ of all cases, and the overall incidence of air embolism - both asymptomatic and symptomatic-most likely lies between 0.4 and $1 \%$ of percutaneous chest biopsies. Low embolized air volume, failure of the air to pass to a critical artery, and rapid resorption of the embolized gas with 100\% oxygen treatment are all factors that most likely contribute to the low incidence of fatal systemic air embolism.

Why worry about asymptomatic cases? Patients have presented with absent or mild, nonspecific symp-

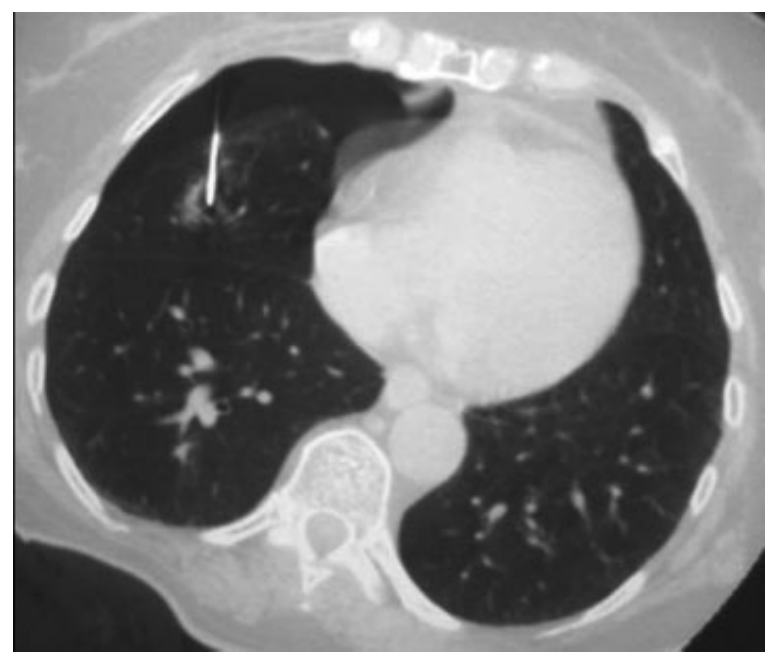

Figure 2 After passage of a 19-gauge introducer needle from an anterior approach through the minor fissure, a followup computed tomography scan showed a moderate anterior pneumothorax. Samples were then attempted using a 20-gauge aspiration needle.

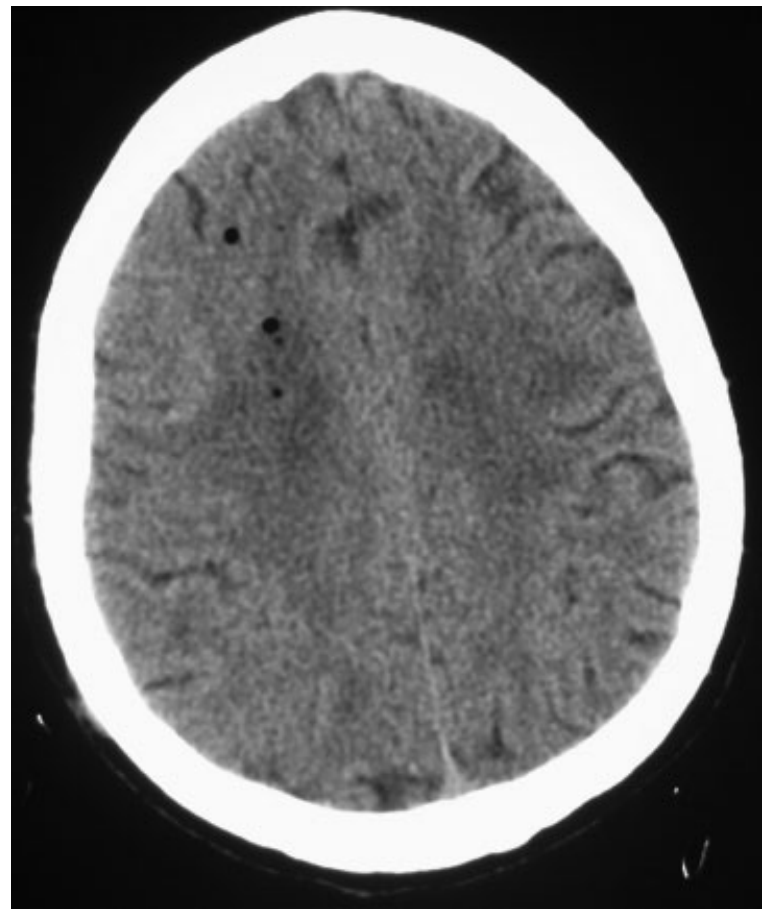

Figure 3 After attempting the first fine need aspiration, the patient became unresponsive. Follow-up head computed tomography shows air in the distribution of the anterior and middle cerebral circulation.

toms at the time of biopsy and later progressed to severe cardiac or permanent neurologic symptoms. ${ }^{4}$ It is currently impossible to predict whether or not patients with asymptomatic or mildly symptomatic systemic air embolism will progress to severe sequelae or death. Because the current literature suggests that a disconcerting percentage of cases are symptomatic, a better understanding of the overall incidence of this entity should be sought, and future prospective studies employing complete head and chest CT after biopsy may be warranted.

\section{Risk Factors and Postulated Mechanisms}

Systemic air embolism can theoretically occur as a result of communication between a pulmonary venous branch and any gas-filled structure, including micro airways, larger bronchi, bullae, cysts, and cavitary lesions. Furthermore, it stands to reason that any additional factor that increases pressure within the offending gas-filled structure during needle manipulation and sample acquisition will facilitate the passage of gas into the vein. Such factors include positive pressure ventilation, straining/ Valsalva, and coughing. Coughing during sample acquisition has been observed in many cases, but this is a common, nonspecific observation during percutaneous chest biopsy and its true association with systemic air embolism is unknown. Exhalation increases airway pressure and inhalation results in the potential for straining at the peak of inhalation (Valsalva), both of which can 
theoretically increase the risk of this complication. Despite these theoretical and anecdotally observed risk factors, systemic air embolism has occurred in cases describing careful adherence to breathing instructions and the absence of coughing, straining or positive pressure ventilation. ${ }^{5}$ On the other hand, most radiologists are keenly aware of the fact that actual adherence to procedure protocols by both physicians and patients may be quite variable and difficult to control, and authors of uncontrolled case studies are disinclined to report deviations from standard protocol.

In most reported cases of systemic air embolism, authors describe standard biopsy technique. The choice of needle size or system has been random in reported cases. In some cases, a single pass with a low-profile $(\leq 22 \mathrm{G})$ biopsy needle was used, ${ }^{6}$ and in other cases, a coaxial system with a $17 \mathrm{G}$ to19G introducer needle was used to facilitate multiple passes. ${ }^{4,7}$ In cases of air embolism after single-pass technique with a low-profile needle, the most likely mechanism is creation of a temporary fistulous tract between an air-filled structure, usually a bronchus, and the pulmonary vein. A review of the existing case studies reveals no particular trend regarding needle size $\mathrm{e}^{6,8}$ or lesion size.

Although "immediate" rapid exchange of the introducer stylet with biopsy needles has been described in reported cases, in our opinion, many of these cases result from an open introducer communicating directly with a pulmonary vein. Inadvertent passage of the needle tip past the lesion and directly into a pulmonary venous branch has been demonstrated. ${ }^{6}$ Safe exchange of device components requires time, creating an inherent risk of air embolism that may ultimately be reduced by the development of valved introducer needles. Precautions to avoid systemic air embolism are outlined in Table 1.

\section{Treatment}

Radiologists should remember that hemodynamic and mental status changes during chest biopsy can have a variety of causes in addition to systemic air embolism, including tension pneumothorax, oversedation, and massive bleeding or pericardial tamponade from injury to a major vessel. Immediate reversal of sedation and administration of $100 \%$ oxygen by bag ventilation through a non-rebreather mask should be performed while steps for performing a complete chest and head $\mathrm{CT}$ are initiated. If necessary, intubation and mechanical ventilation should be initiated. Rescue therapies for systemic air embolism were developed largely for treatment of this complication after coronary angiography, surgery, and positive pressure ventilation. Hyperbaric oxygen therapy has emerged as the best treatment option. If the CT shows systemic air embolism, immediate arrangements for transfer to a hyperbaric oxygen chamber should be initiated. A stat 12-lead electrocardiogram should be obtained to evaluate for developing signs of acute myocardial infarction, including ST segment elevation and arrhythmias. A full code should be considered in all cases along with consideration of pharmacological agents to increase coronary perfusion.

Patient positioning remains a subject of controversy. Various authors have recommended placement of the patient in the left lateral decubitus ${ }^{8-10}$ and Trendelenberg ${ }^{11}$ positions to prevent progression of gas through the left ventricular outflow tract and to the intracranial or coronary circulation. Others have recommended maintaining supine positioning ${ }^{12}$ to minimize patient movement and further migration of air. The hemodynamic benefits of each position have been postulated, but the frequency of this complication and the details provided in reported cases are insufficient to date to resolve this issue. Many practitioners believe that the most important, and potentially the only efficacious, immediate measure is supplementation with $100 \%$ oxygen by non-rebreather mask or intubation and, if necessary, bag or mechanical ventilation, followed by the initiation of hyperbaric oxygen treatment. Hyperbaric oxygen lasting hours to days has been successfully performed as a rescue therapy in several described cases. This treatment decreases intravascular bubble size, increases oxygen diffusion into tissues, and reduces the nitrogen content of the bubbles, facilitating their eventual resorption. At the conclusion of hyperbaric oxygen therapy, complete resorption of intravascular gas has been observed in most cases by cross-sectional imaging. ${ }^{4,5}$

\section{Remaining Questions}

Should the entire chest be scanned routinely after performing CT-guided percutaneous chest biopsy? Hiraki et $\mathrm{al}^{4}$ found 3 asymptomatic systemic air embolism cases out

Table 1 Precautions to Avoid Air Embolism

- Patients should be coached prior to the procedure that they should suspend respiration when instructed

(during any needle manipulation). This instruction also minimizes the risk of pneumothorax and nontarget puncture.

- The period during which an introducer is open to air should be kept to an absolute minimum when using coaxial technique.

- Prior to obtaining samples, a scan should be performed to verify the final position of the introducer needle. Computed

tomography fluoroscopy may be helpful to ensure proper tip positioning.

- The gas-filled components of a cystic or cavitary lesion should be avoided if possible.

- Large pulmonary venous branches should be avoided if possible. 
of 860 biopsies by performing postbiopsy complete chest CTs. In fact, their overall results show that in $50 \%$ of cases of systemic air embolism, the systemic air was located outside the target area and would only be discovered by performance of a complete chest CT. The value of performing routine, complete chest $\mathrm{CT}$ s after chest biopsies remains a subject of debate.

Should hyperbaric oxygen be prescribed for asymptomatic systemic air embolism? Clearly, the $20 \%$ of systemic air embolism patients that become symptomatic require treatment as soon as possible. On the other hand, patients with an asymptomatic air embolism may be at high risk for developing the devastating sequelae. Asymptomatic patients have progressed to permanent sequelae or death. ${ }^{4}$ Does moving or transferring asymptomatic patients lead to distal air migration? Is there sufficient time to initiate rescue hyperbaric oxygen therapy after patients have become symptomatic and the diagnosis of air embolism has been confirmed by imaging studies? Although delayed hyperbaric therapy has proven efficacious in isolated cases, ${ }^{13}$ case studies have reported deaths from air embolism when transfer to an outside facility for hyperbaric oxygen treatment was required. ${ }^{5}$ Prophylactic treatment of asymptomatic patients is not without risks because hyperbaric oxygen has been associated with generalized seizures and rupture of the middle ear, cranial sinuses, and lungs. ${ }^{14}$ An argument can be made that the potentially devastating sequelae of systemic air embolism make the risks of hyperbaric oxygen therapy more palatable for at least some asymptomatic patients.

\section{SUGGESTED READINGS}

Sinner WN. Complications of percutaneous transthoracic needle aspiration biopsy. Acta Radiol Diagn (Stockh) 1976;17(6): 813-828
Richardson CM, Pointon KS, Manhire AR, Macfarlane JT. Percutaneous lung biopsies: a survey of UK practice based on 5444 biopsies. Br J Radiol 2002;75(897):731-735

Tomiyama N, Yasuhara Y, Nakajima Y, et al. CT-guided needle biopsy of lung lesions: a survey of severe complication based on 9783 biopsies in Japan. Eur J Radiol 2006;59(1):60-64

Hiraki T, Fujiwara H, Sakurai J, et al. Nonfatal systemic air embolism complicating percutaneous CT-guided transthoracic needle biopsy: four cases from a single institution. Chest 2007;132(2):684-690

Bou-Assaly W, Pernicano P, Hoeffner E. Systemic air embolism after transthoracic lung biopsy: A case report and review of literature. World J Radiol 2010;2(5):193-196

Mokhlesi B, Ansaarie I, Bader M, Tareen M, Boatman J. Coronary artery air embolism complicating a CT-guided transthoracic needle biopsy of the lung. Chest 2002;121(3):993-996

Hsi DH, Thompson TN, Fruchter A, Collins MS, Lieberg OU, Boepple H. Simultaneous coronary and cerebral air embolism after CT-guided core needle biopsy of the lung. Tex Heart Inst J 2008;35(4):472-474

Ghafoori M, Varedi P. Systemic air embolism after percutaneous transthorasic needle biopsy of the lung. Emerg Radiol 2008; 15(5):353-356

Kau T, Rabitsch E, Celedin S, Habernig SM, Weber JR, Hausegger KA. When coughing can cause stroke-a casebased update on cerebral air embolism complicating biopsy of the lung. Cardiovasc Intervent Radiol 2008;31(5): 848-853

Tolly TL, Feldmeier JE, Czarnecki D. Air embolism complicating percutaneous lung biopsy. AJR Am J Roentgenol 1988;150(3): $555-556$

Arnold BW, Zwiebel WJ. Percutaneous transthoracic needle biopsy complicated by air embolism. AJR Am J Roentgenol 2002;178(6):1400-1402

Muth CM, Shank ES. Gas embolism. N Engl J Med 2000; 342(7):476-482

Ohashi S, Endoh H, Honda T, Komura N, Satoh K. Cerebral air embolism complicating percutaneous thin-needle biopsy of the lung: complete neurological recovery after hyperbaric oxygen therapy. J Anesth 2001;15(4):233-236

Tibbles PM, Edelsberg JS. Hyperbaric-oxygen therapy. N Engl J Med 1996;334(25):1642-1648 